\title{
A microprocessor-based real-time BASIC random interstimulus interval generator with different probability density functions
}

\author{
NAOYUKI OSAKA \\ Otemon-Gakuin University, Ibaraki, Osaka, 567 Japan
}

\begin{abstract}
A low-cost random interstimulus interval (ISI) generator with different probability density functions is described. The frequency histograms of the output ISIs are uniformly, normally, exponentially, and binary distributed functions.
\end{abstract}

Recent developments in microprocessor technology and related peripheral interfacing (PIA) technology make real-time randomization of an external stimulus device inexpensive (see Brown \& Deffenbacher, 1978; Kashinsky, Romanczyk, \& Treiber, 1979; Milkman, Shapley, \& Schick, 1978; Perera, 1978; Polson, 1978; Santa \& Streit, 1978; Sidowski, 1978).

A low-cost circuit for a quasirandom interstimulus interval (ISI) generator using shift registers has been described by Damashek (1976) and Osaka (1978a, 1978b). Randomization techniques based on pseudorandom number generation (Jansson, 1966) have been written in assembly (Grieser, 1977; for 8080 and 6800 CPUs), BASIC (Lehman, 1977; Nilsson, 1978; Robertson, 1977), and FORTRAN (Green, 1977).

Using the Commodore PET 2001 microcomputer as a random number generator, visual and auditory stimuli can easily be signaled with random ISIs having different probability density functions.

In the system described here, the PET communicates with two LEDs and an 8-ohm speaker via 6522 PIA (VIA) $\mathrm{I} / \mathrm{O}$ ports, as shown in Figure 1 (see McLean, 1978; Yob, 1979a, 1979b, for the PET's port handling techniques; see also Commodore Business Machines, Inc., 1978, Note 1; PET User Group, 1978). Only PA5, PA6, and CB2 ports are defined as output modes. Figure 2 shows a general flow chart for the random ISI generator written in BASIC, as shown in Figure 3.

\section{PROGRAMMING}

As shown in Figures 2 and 3, after initialization (Lines 10-30), the experimenter inputs the tone duration, ISI factor, random number generator subroutine select (Lines 32-50), print select (to the 9-in. CRT), and ISI unit select (either $100-\mathrm{msec}$ or $1-\mathrm{sec}$ step ISI) sequentially. After finding these input values, the PET generates random ISIs with selected random ISI fre-

This work was supported in part by Grant 401059 from the Ministry of Education, Japan.

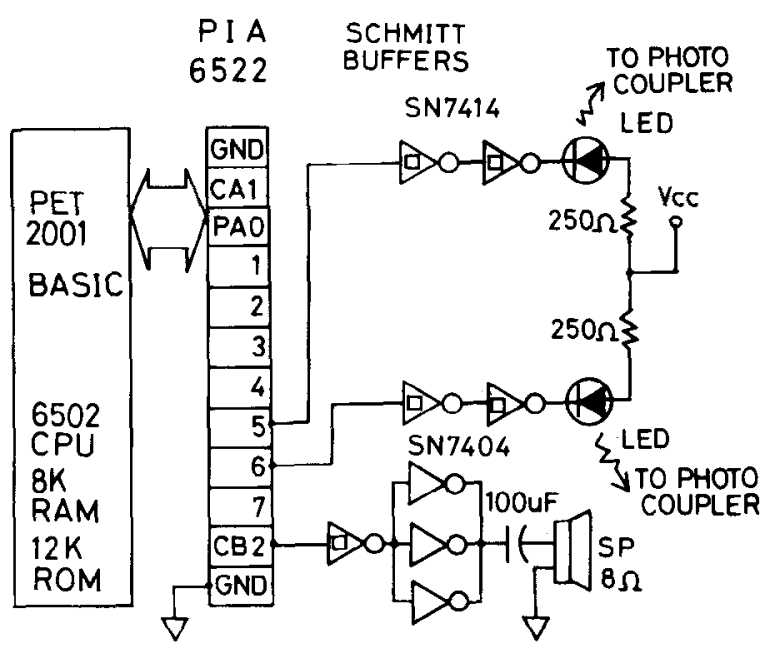

Figure 1. An interfacing logic for the random ISI generator.

quency: uniformly (UNIF), normally (NORM), exponentially (EXP), and binary (BIN) distributed frequency and randomization (RNDMZ) without replacement.

\section{UNIF (Lines 1000-1140)}

Using PET BASIC, uniformly distributed random numbers ranging from 1 to 10 can be obtained easily (Line 1050). The scale factor YY (Line 1053) determines the size of the mean ISI.

\section{NORM (Lines 2000-2165)}

Using a central limit theorem, the random numbers with normal distribution are obtained from a series of uniform random numbers by the formula $\mathrm{R}=[\mathrm{T} / \mathrm{L}-.5] / \mathrm{SQR}\left[1 /\left(12^{*} \mathrm{~L}\right)\right]$, where $\mathrm{T}$ indicates a sum of uniform random numbers up to $\mathrm{L}$. If $\mathrm{L}=12$, the above formula is simplified as [T-6]. The size of $L$ and $N$ (sample size) determines normality of the distribution function. 


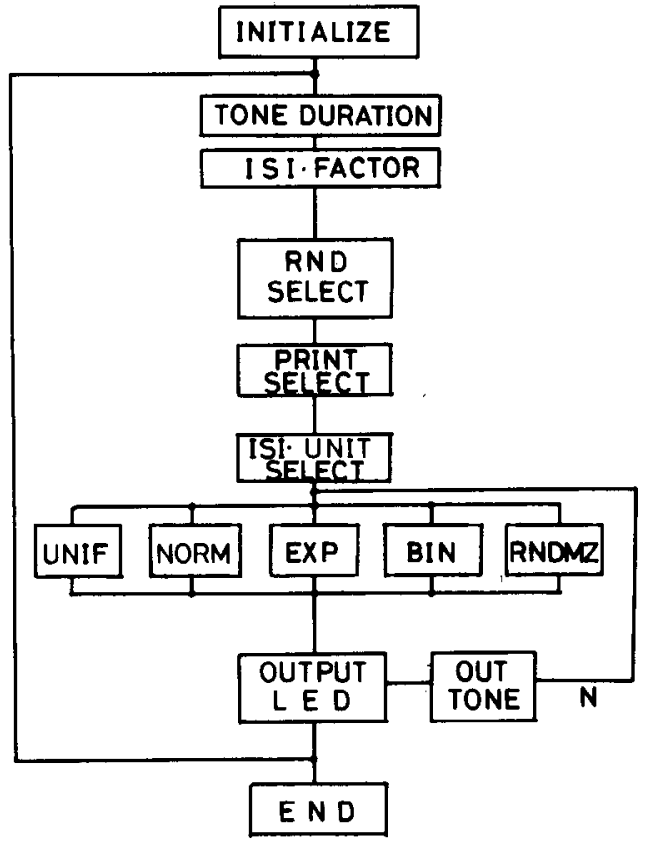

Figure 2. A general flow chart for the random ISI generator.

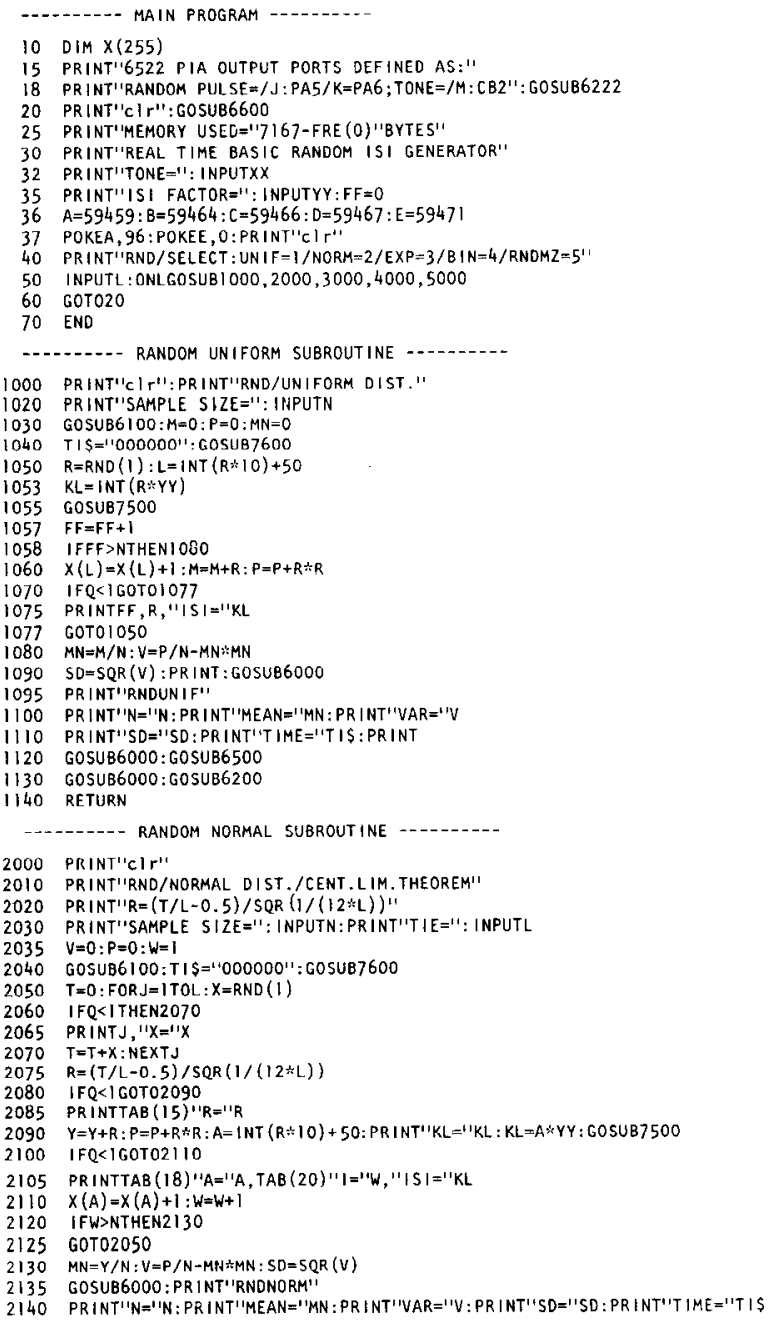

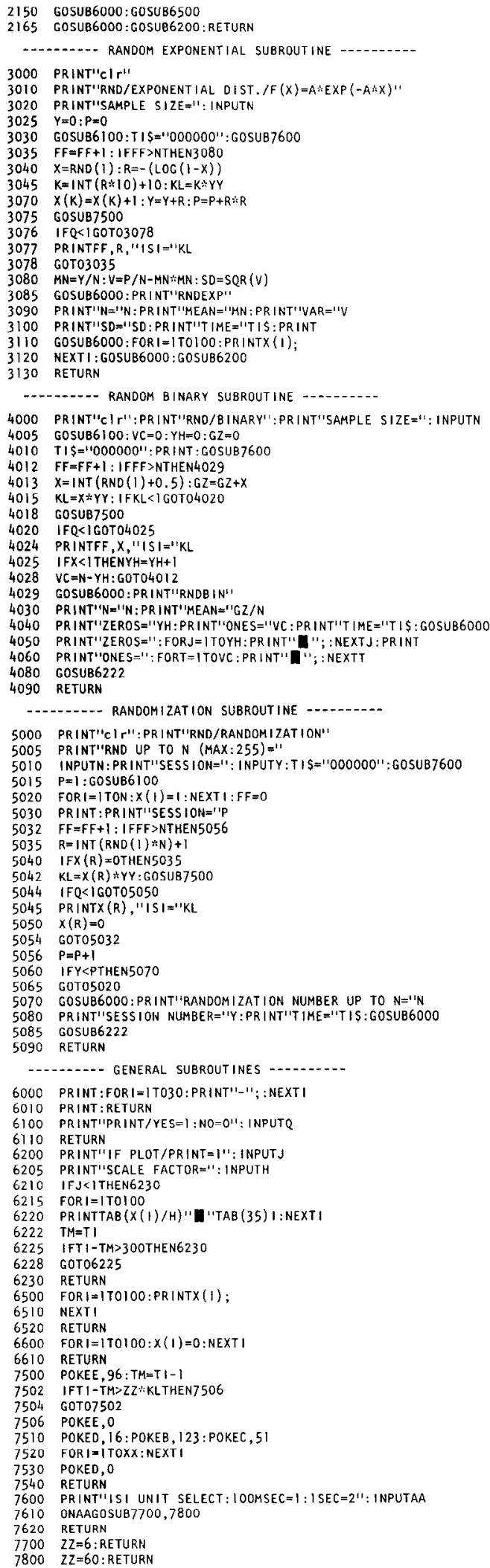

Figure 3. An example program for real-time BASIC random ISI generator. Random ISls having uniform (UNIF), normal (NORM), exponential (EXP), binary (BIN), and randomized (RNDMZ) distribution can be generated. 


\section{EXP (Lines 3000-3130)}

The exponentially distributed random numbers using the method of inverse function are generated by the formula $\mathrm{R}=-[\operatorname{LOG}(1-\mathrm{X})]$. (A log transformation is used on each uniform random number.)

\section{BIN (Lines 4000-4090)}

These binary $(0$ or 1$)$ numbers result from the formula $X=\operatorname{INT}(\operatorname{RND}(1)+.5)$, where $\operatorname{RND}(1)$ generates uniform random numbers.

\section{RNDMZ (Lines 5000-5090)}

This subroutine randomizes numbers up to $\mathrm{N}$ without replacement (Lines 5035-5040). The program appears suitable for real-time BASIC laboratory automization (BLA), since the output signals can be applicable to drive external devices such as pulse motors up to $\mathrm{Yth}$ session. Careful attention is required because the time to draw and reject terms is slightly longer for the later trials due to the sampling without replacement algorithm. This can introduce unwanted variation in an ISI.

Mean, variance, and standard deviation are printed out. In addition, the dimension statement, such as $\mathrm{X}(\mathrm{I})=\mathrm{X}(\mathrm{I})+1$, prints out the graphic output of each distribution if required.

In the general subroutines (Lines 6000-7800), the PET prints out the output ISI distribution on print request following input of a scale factor value (Lines 6200-6230). It returns to the main program after $5 \mathrm{sec}$ (Lines 6225-6228). In the statements from 7500 to 7540 , the 1/O port PA5 and PA6 of the 6522 PIA are defined as output mode (POKE E, ${ }^{1}$ 96[ $[=64+32]$ ), and the formula $[\mathrm{TI}-\mathrm{TM}>\mathrm{ZZ} * \mathrm{KL}]^{2}$ determines the ISIs, where $Z Z$ and $K L$ show the ISI unit select parameter and the ISI factor, respectively. Immediately after the end of the ISI, a $1-\mathrm{kHz}$ tone is sounded for a given duration (Lines 7510-7530).

\section{OUTPUT}

Figure 4 shows an example program for calibrating

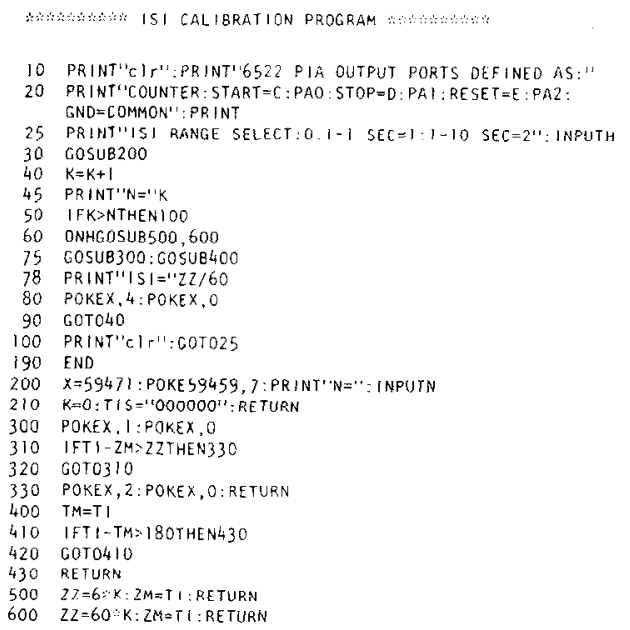

Figure 4. An example program for calibrating ISI using digital precision counter.

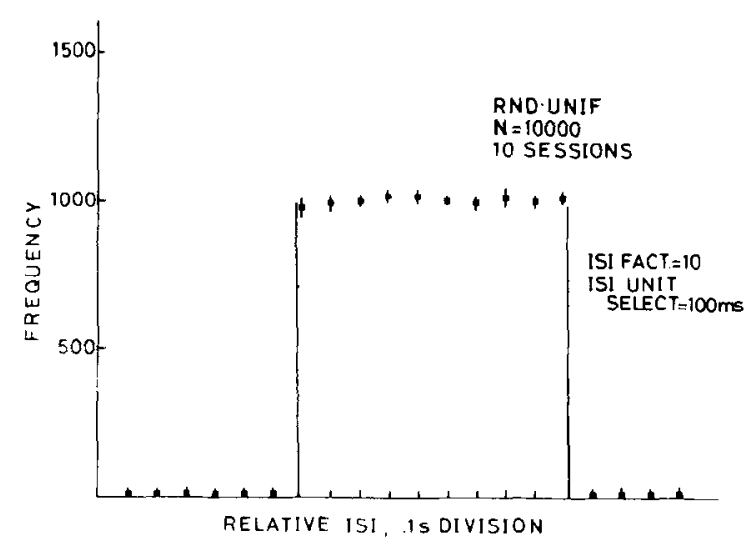

Figure 5. An output ISI histogram of the UNIF distribution.

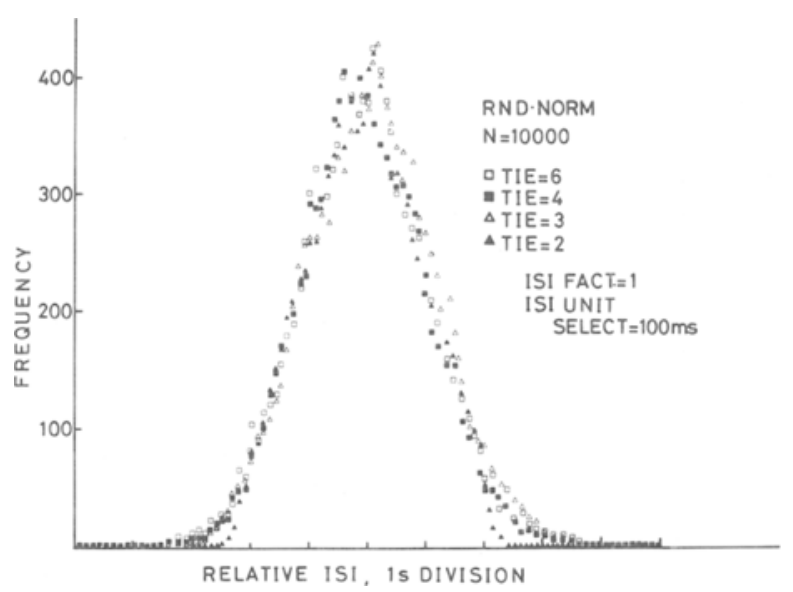

Figure 6. An output ISI histogram of the NORM distribution.

ISI using a digital precision counter incorporating a $100-\mathrm{kHz}$ crystal oscillator. Gating of the counter is made via PA0 (start pulse), PA1 (stop pulse), and PA2 (reset pulse) ports. Since the unit of the TI parameter of the PET is $1 / 60 \mathrm{sec}$, the error ratio is less than $1 \%$ for each time setting. Figures $5,6,7$, and 8 show output ISI histograms for UNIF, NORM, EXP, and BIN, respectively.

\section{UNIF}

In Figure 5, when $\mathbf{N}=10,000$ for each session, a uniformly distributed histogram is obtained. The vertical bar shows the standard deviation.

\section{NORM}

Figure 6 clearly shows a normally distributed frequency histogram. Note that the larger the size of the $\operatorname{TIE}(=\mathrm{L})$, the shallower the tail of the normal distribution curve.

\section{EXP}

Figure 7 shows an exponentially distributed frequency histogram. The vertical bar shows the standard deviation. 


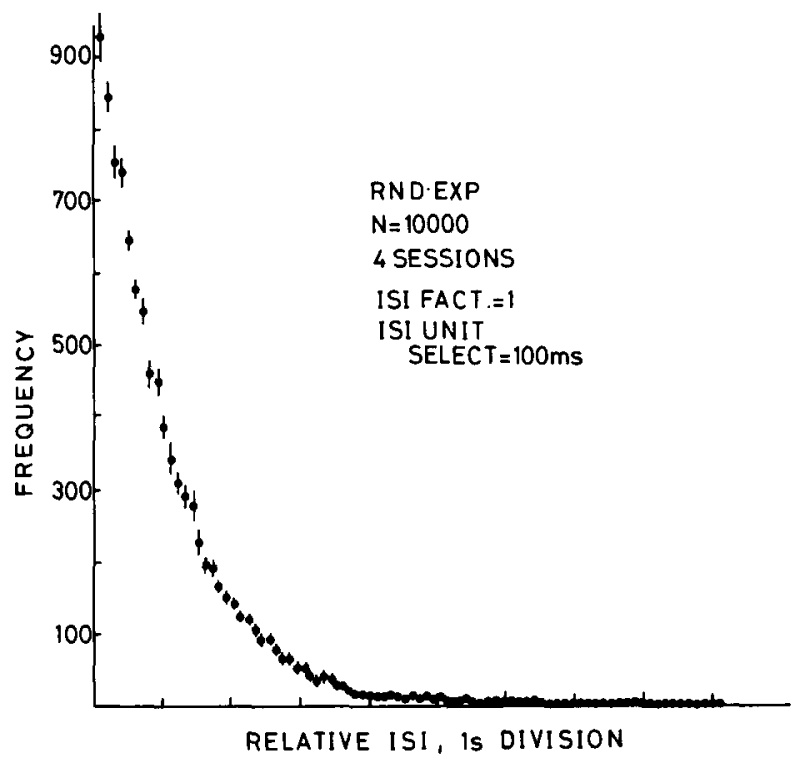

Figure 7. An output ISI histogram of the EXP distribution.

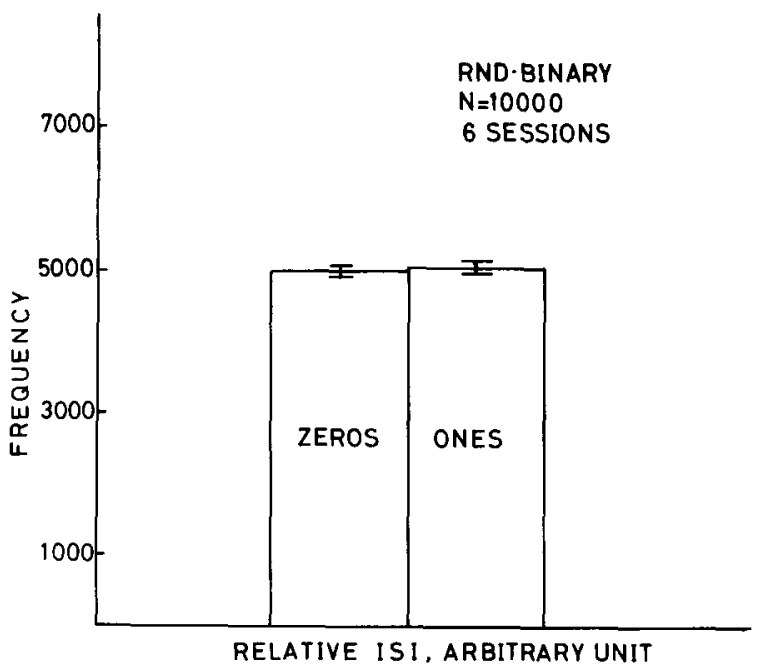

Figure 8. An output ISI histogram of the BIN distribution.

\section{BIN}

Figure 8 indicates uniformly distributed zeros and ones over six sessions. The vertical bar shows the standard deviation.

\section{RNDMZ}

When $\mathrm{N}=255$, randomization without replacement requires $403 \mathrm{sec}$ for each session (ISI unit select $=1$ ).

In general, the random ISI frequency histograms show the random ISI generator to be useful for realtime BASIC laboratory automization (BLA).

\section{REFERENCE NOTE}

1. Commodore Business Machines, Inc. MCS6522 versatile interface adaptor. Preliminary data sheet, November 1977.

\section{REFERENCES}

Brown, E. L., \& Deffenbacher, K. Microcomputers big and little: Selecting a low-cost laboratory computer network. Behavior Research Methods \& Instrumentation, 1978, 10, 241-245.

Commodore Business Machines, Inc. PET communication with the outside world. Palo Alto, Calif: Author, 1978.

DAMAShE K, M. Shift register with feedback generates white noise. Electronics, 1976, 49, 107-109.

GreEN, B. F., JR. FORTRAN subroutines for random sampling without replacement. Behavior Research Methods \& Instrumentation, 1977, 9, 559.

Grieser, D. Pseudorandom number generator. Byte, 1977, 2, 218.

JANSSON, B. Random number generators. Stockholm: Almqvist \& Wiksell, 1966.

Kashinsky, W. M., Romanczyk, R. G., \& Treiber, A. The presentation of visual stimuli: An inexpensive microcomputerbased system. Behavior Research Methods \& Instrumentation, $1979,11,64-66$.

LeHman, R. S. An improved BASIC program to produce random samples without replacement. Behavior Research Methods \& Instrumentation, 1977, 9, 555-556.

McLean, R. S. The Commodore PET: Using personal computers for experimental control. Behavior Research Methods \& Instrumentation, 1978, 10, 468-473.

Milkman, N., Shapley, R., \& Schick, G. Experimental applications: A microcomputer-based visual stimulator. Behavior Research Methods \& Instrumentation, 1978, 10, 539-545.

Nilsson, T. H. Randomization without replacement using replacement without losing your place. Behavior Research Methods \& Instrumentation, 1978, 10, 419.

OsAKA, N. An inexpensive quasirandom interpulse interval generator. Behavior Research Methods \& Instrumentation, $1978,10,18-20$. (a)

Osaka, N. Pseudo-random pulse generator using shiftregisters. Japanese Journal of Psychology, 1978, 49, 99-102. (b)

Perera, T. B. A versatile microcomputer-based multiple-field tachistoscope. Behavior Research Methods \& Instrumentation. 1978, 10, 546-547.

PET User Group. PET user notes (No. 6). South Bound Brook, N.J: Author, 1978.

Pouson, P. G. Microprocessors: Their impact on real-time computing in psychology. Behavior Research Methods \& Instrumentation, 1978, 10, 463-467.

RoBERTSON, S. A. A BASIC program to randomly generate numbers of a weighted set. Behavior Research Methods \& Instrumentation, 1977, 9, 547-548.

Santa, J. L., \& Streit, P. A. A microprocessor reaction time laboratory. Behavior Research Methods \& Instrumentation, $1978,10,352-355$.

SidowsKi, J. B. Microcomputers in psychology. Behavior Research Methods \& Instrumentation, 1978, 10, 463-467.

Yoв, G. PET techniques explained. Kilobaud, 1979, 25, 82-90. (a)

YoB, G. PET user port cookbook. Kilobaud, 1979, 27, 62-73. (b)

\section{NOTES}

1. Decimal coded addressed locations: $A=$ direction register; $\mathbf{B}=$ Timer 2 register $; C=$ shift register; $D=$ auxiliary control register; $\mathrm{E}=$ output register without handshaking.

2. Since PET's TI parameter is a counter of $1 / 60$-sec steps, a statement such as [TI*300] determines a 5-sec lapse of the time.

(Received for publication April 9, 1979; revision accepted September $21,1979$. 